\title{
Pulmonary artery size as a predictor of outcomes in idiopathic pulmonary fibrosis
}

\author{
Stephanie Shin ${ }^{1}$, Christopher S. King ${ }^{2}$, Nitin Puri ${ }^{3}$, Oksana A. Shlobin ${ }^{2}$, \\ A. Whitney Brown ${ }^{2}$, Shahzad Ahmad ${ }^{2}$, Nargues A. Weir ${ }^{2}$ and Steven D. Nathan ${ }^{2}$ \\ Affiliations: \\ ${ }^{1}$ Dept of Pulmonary and Critical Care, University of California San Diego, San Diego, CA, USA. \\ ${ }^{2}$ Advanced Lung Disease and Lung Transplant Program, Dept of Medicine, Inova Fairfax Hospital, Falls \\ Church, VA, USA. \\ ${ }^{3}$ Dept of Pulmonary and Critical Care, Dept of Medicine, Inova Fairfax Hospital, Falls Church, VA, USA.
}

Correspondence:

Steven D. Nathan, Advanced Lung Disease and Transplant Program, Inova Heart and Vascular Institute, 3300 Gallows Road, Falls Church, VA 22042, USA.

E-mail: steven.nathandinova.org

ABSTRACT IPF patients have heightened propensity for pulmonary hypertension, which portends a worse outcome. Presence of pulmonary hypertension may be reflected in an enlarged pulmonary artery. We investigated pulmonary artery size measured on high-resolution computed tomography (HRCT) as an outcome predictor in IPF.

We retrospectively reviewed all IPF patients evaluated at a tertiary-care centre between 2008 and 2013. Pulmonary artery and ascending aorta diameters were measured from chest HRCT with pulmonary artery: ascending aorta diameter (PA:A) ratio calculations. Outcome analysis defined by either death or lung transplant based on pulmonary artery size and PA:A ratio over 60 months was performed. Independent effects of different variables on overall outcomes were evaluated using the Cox proportional hazards model.

98 IPF patients with available HRCT scans had a mean pulmonary artery diameter and PA:A ratio of $32.8 \mathrm{~mm}$ and 0.94 , respectively. Patients with a PA:A ratio $>1$ had higher risk of death or transplant compared with a PA:A ratio $\leqslant 1(\mathrm{p}<0.001)$. A PA:A ratio $>1$ was also an independent predictor of outcomes in unadjusted and adjusted outcomes analyses (hazard ratio 3.99, $\mathrm{p}<0.001$ and hazard ratio 3.35, $\mathrm{p}=0.002$, respectively).

A PA:A ratio $>1$ is associated with worse outcomes in patients with IPF. HRCT PA:A ratio measurement may assist in risk stratification and prognostication of IPF patients.

@ERSpublications

Pulmonary artery measurements on imaging can be utilised for risk stratification and prognostication in IPF http://ow.ly/WNO2h

Editorial comment in: Eur Respir J 2016; 47: 1318-1320.

This article has supplementary material available from erj.ersjournals.com

Received: Sept 132015 | Accepted after revision: Dec 212015 | First published online: Feb 042016

Conflict of interest: Disclosures can be found alongside the online version of this article at erj.ersjournals.com

Copyright @ERS 2016 


\section{Introduction}

Idiopathic pulmonary fibrosis (IPF), the most common form of the idiopathic interstitial pneumonias, is a chronic, progressive fibrotic disease that is associated with significant morbidity and mortality. The clinical course of IPF is generally characterised by progressive deterioration with a median survival of 2.5-4 years [1]. The course of IPF in individual patients is notoriously difficult to predict. Prognostication has typically been based on pulmonary function tests, specifically forced vital capacity (FVC) and single-breath diffusing capacity of the lung for carbon monoxide (DLCO) [2]. However, other characteristics, including age, gender and body mass index (BMI), may all influence outcomes. Indeed, a composite of variables in the form of the GAP index (based on gender, age and two lung physiology variables (FVC and DLCO)) has gained recognition as a more accurate predictor of outcomes [3]. IPF is a disease that is characterised by a high prevalence of comorbidities, including pulmonary hypertension, coronary artery disease, gastro-oesophageal disease and obstructive sleep apnoea [2, 4]. These comorbidities may also be associated with worse outcomes; of these, pulmonary hypertension has the strongest association with functional impairment and survival [5-7]. Aside from DLCO, which has a weak correlation with pulmonary hypertension, the GAP score does not incorporate elements that capture the impact of complicating pulmonary hypertension.

Given the independent prognostic significance of pulmonary hypertension in IPF, clinical suspicion and early diagnosis would appear to be essential. For example, the development of pulmonary hypertension in patients with IPF might be an indication for listing for lung transplantation as recommended in the most recent international consensus guidelines [8]. The presence of pulmonary hypertension may help risk-stratify patients for clinical trials with the pulmonary hypertension itself being a target of such trials. There are a number of parameters that might suggest the presence of pulmonary hypertension, including "disproportionate" dyspnoea, elevated brain natriuretic peptide and 6-min walk test variables, including short distance walked, increased desaturation and a low pulse rate recovery. The best screening tool for pulmonary hypertension is transthoracic echocardiography; however, it has a greater tendency to be inaccurate in patients with advanced lung disease $[9,10]$.

An ancillary, noninvasive screening tool would facilitate more accurate risk stratification and allow more judicious use of the gold standard right heart catheterisation (RHC). Evidence of pulmonary hypertension on chest computed tomography (CT) could represent a readily available tool to more accurately predict the presence of pulmonary hypertension. To date, studies on the ability of chest CT to predict pulmonary hypertension have variably included an evaluation of the pulmonary artery diameter, the pulmonary artery:ascending aorta diameter (PA:A) ratio or measurements of segmental vessels. Indeed, the association between pulmonary artery size and prevalent pulmonary hypertension in fibrotic lung disease has yielded mixed results [11-14]. However, the association between pulmonary artery dilation on CT and clinical outcomes in IPF has, to the best of our knowledge, not been assessed, while recent studies in chronic obstructive pulmonary disease (COPD) have demonstrated the ability of the ratio of the pulmonary artery to the aorta to predict exacerbations and mortality $[15,16]$.

In this study, we sought to establish whether an independent relationship exists between pulmonary artery size based on CT measurements and outcomes in IPF patients.

\section{Methods}

Study design

Patients with IPF evaluated in an Advanced Lung Disease clinic between August 2008 and 2013 with high-resolution CT (HRCT) scans were identified. The diagnosis of IPF was made based on current standard of care at the time of evaluation and referred at varying stages of disease for management $[17,18]$. The HRCT scans were reviewed in a blinded and independent fashion by three of the authors (S. Shin, C.S. King and N. Puri). The pulmonary artery segment was measured at its bifurcation with the largest ascending aorta diameter measured at the corresponding level with the PA:A ratio calculated from these measurements [16]. The presence of aortic aneurysms was also evaluated with threshold aortic diameters accounting for the effects of age and gender. Specifically, the upper limits of normal were defined as 35.6, 38.3 and $40 \mathrm{~mm}$ for females and 37.8, 40.5 and $42.6 \mathrm{~mm}$ for males in the age ranges 20-40, 41-60 and $>60$ years, respectively [19]. When available, pulmonary arterial pressure (PAP) measurements from the RHC were also obtained for analysis.

The means of the three independent measurements for the pulmonary artery and the PA:A ratio were used for data analysis. Demographic data collated included age, BMI, gender and pulmonary function tests.

Our primary endpoint was transplant-free survival, which was calculated from the date of the chest CT until either death or transplant, whichever event occurred first. For outcome analyses based on pulmonary function tests, time zero for each patient was calculated from the date of the pulmonary function tests. The study was approved by the Inova Institutional Review Board (IRB15-1955). 


\section{Statistics}

Continuous data are presented as mean or as median (range), while categorical data are presented as frequencies and percentages. Parametric statistics were utilised for data confirmed to have a normal distribution. The intra-class correlation coefficient was used to determine the level of agreement among the three readers for continuous variables of the pulmonary artery and the PA:A ratio. Comparisons of measurements between groups were performed using unpaired t-tests. Pearson's correlation was utilised for comparison of the pulmonary diameter and PA:A ratio to the mean PAP (mPAP) and FVC. The primary outcome analyses of death or transplant were demonstrated with Kaplan-Meier survival curves and log-rank testing. Cox proportional hazard regression was performed to assess the association between PA:A ratio and lung transplant or death. The GAP index was calculated for each patient, and included in the regression model to control for potential confounding factors of age, gender, DLCO \% predicted and FVC \% predicted [3]. Hazard ratios were attained from the Cox analyses. All calculations were performed utilising SAS software version 9.2 (SAS Institute, Cary, NC, USA) and IBM SPSS software version 20.0 (IBM, Armonk, NY, USA).

\section{Results}

There were 98 IPF patients identified who were evaluated over the 5-year period in whom chest HRCT images were available. The demographics and pulmonary function of the group are shown in table 1 . The patients were divided into two groups based on those with a PA:A ratio $\leqslant 1$ and a PA:A ratio $>1$. The baseline characteristics were not significantly different with the exception of the FVC \% predicted, forced expiratory volume in $1 \mathrm{~s} \%$ predicted and DLCO \% predicted. The GAP index was higher in those with PA:A ratio $>1(p=0.02)$.

The mean (range) pulmonary artery and ascending aorta diameters were 32.8 (20.9-45.4) and 35.0 (24.9$47.8) \mathrm{mm}$, respectively. The mean PA:A ratio was 0.94 with an intra-class correlation coefficient amongst the three readers demonstrating excellent agreement $(\mathrm{R}=0.79,95 \% \mathrm{CI} 0.68-0.86, \mathrm{p}<0.001)$. The correlation between FVC \% predicted and PA:A ratio demonstrated a negative correlation $(r=-0.31, p=0.002)$. The RHC data were available for 53 patients with a mean CT to RHC time interval of 7.2 months, of which $66 \%(n=35)$ of patients had mPAP $<25 \mathrm{mmHg}$. The overall correlation between mPAP and PA:A ratio was 0.35 (online supplementary table $\mathrm{S} 1$ ). The correlation was stronger $(\mathrm{r}=0.57)$ for the smaller subset of patients $(\mathrm{n}=27)$ with RHC data within 3 months of their chest CT (online supplementary table S2). A similar correlation between mPAP and pulmonary artery diameter size was also noted, with strengthening of this correlation for the subset of patients with a shorter time interval between the two studies (online supplementary table S3).

Table 2 shows the mean ascending aorta and pulmonary artery diameter stratified by PA:A ratio $\leqslant 1$ and $\mathrm{PA}: \mathrm{A}$ ratio $>1$. The mean pulmonary artery diameter for PA:A ratio $\leqslant 1$ and PA:A ratio $>1$ was 31.1 and

TABLE 1 Patient characteristics stratified by pulmonary artery:ascending aorta diameter (PA:A) ratio

\begin{tabular}{|c|c|c|c|c|}
\hline & All & $\mathrm{PA}: \mathrm{A}$ ratio $\leqslant 1$ & PA:A ratio >1 & p-value \\
\hline Subjects $n$ & 98 & 67 & 31 & \\
\hline Age years & 67 & 67 & 67 & 0.84 \\
\hline BMI $\mathrm{kg} \cdot \mathrm{m}^{-2 \#}$ & 29.8 & 29.6 & 30 & 0.52 \\
\hline Female $n$ & 18 & 12 & 6 & 0.87 \\
\hline FVC \% pred & 64 & 67 & 57 & 0.01 \\
\hline FEV $_{1} \%$ pred & 71 & 74 & 64 & 0.02 \\
\hline $\mathrm{FEV}_{1} / \mathrm{FVC} \%{ }^{\uparrow}$ & 83 & 83 & 84 & 0.28 \\
\hline TLC \% pred ${ }^{+}$ & 64 & 65 & 57 & 0.05 \\
\hline DLco \% pred & 41 & 45 & 34 & $<0.01$ \\
\hline GAP index & 4.29 & 4.01 & 4.87 & 0.02 \\
\hline CAD risk factors ${ }^{\S}$ & 2.30 & 2.36 & 2.16 & 0.46 \\
\hline
\end{tabular}

Data presented as means, unless otherwise stated. BMI: body mass index; FVC: forced vital capacity; FEV1: forced expiratory volume in $1 \mathrm{~s}$; TLC: total lung capacity; DLCO: diffusing capacity of the lung for carbon monoxide; GAP index: scoring system based on gender, age and two lung physiology variables (FVC and DLCO); CAD: coronary artery disease. Significance tests for comparisons between groups based on two-sample independent t-tests for continuous patient characteristics and Pearson's Chi-squared tests for categorical patient characteristics. \#: BMI missing patient data $n=4$; П: FEV $1 / F V C$ missing patient data $n=4$; ${ }^{+}$: TLC missing patient data $n=7 ;{ }^{\S}$ : risk factors: hypertension, hyperlipidaemia, diabetes mellitus, smoking and family history CAD risk factors (1 point for each risk factor). 
TABLE 2 HRCT measurements stratified by pulmonary artery:ascending aorta (PA:A) diameter ratio

\begin{tabular}{lccc} 
& PA:A ratio $\leqslant 1$ & PA:A ratio $>\mathbf{1}$ & p-value \\
\hline Subjects $\mathbf{n}$ & 67 & 31 & \\
Ascending aorta diameter $\mathbf{m m}$ & $35.6(31.5-39.7)$ & $33.7(29.9-37.5)$ & 0.02 \\
Pulmonary artery diameter $\mathbf{m m}$ & $31.1(27.7-34.5)$ & $36.3(32.5-40.1)$ & $<0.001$ \\
\hline
\end{tabular}

Data are presented as mean $(95 \% \mathrm{CI})$, unless otherwise stated.

$36.3 \mathrm{~mm}$, respectively. The mean ascending aorta diameter for PA:A ratio $\leqslant 1$ and PA:A ratio $>1$ was 35.6 and $33.7 \mathrm{~mm}$, respectively. There were six patients with aortic dilation/aneurysm $(\mathrm{n}=5$ in the PA:A ratio $\leqslant 1$ group and $n=1$ for the PA:A ratio $>1$ group). The mean pulmonary artery diameters for those with aortic dilations/aneurysms were 33.6 and $45 \mathrm{~mm}$ for PA:A ratio $\leqslant 1$ and PA:A ratio $>1$, respectively.

The Kaplan-Meier outcomes analysis of time to death or transplant based on pulmonary artery size and PA:A ratio are demonstrated in figures 1 and 2. Patients above the median pulmonary artery diameter for the cohort $(>32.3 \mathrm{~mm})$ had worse outcomes compared with those below the median (figure $1 ; \mathrm{p}=0.03$ ). Those with PA:A ratio $>1$ also demonstrated worse outcomes compared to those with PA:A ratio $\leqslant 1$ (figure 2; $\mathrm{n}=13$ lung transplantation and $\mathrm{n}=18$ death, $\mathrm{p}<0.001$ ). A subgroup Kaplan-Meier outcomes analysis excluding the six patients with aortic dilations/aneurysms was also performed and it demonstrated the same outcome difference $(\mathrm{p}<0.001)$.

The unadjusted and adjusted hazard ratio calculations using the Cox proportional hazard analysis adjusting for the GAP index with PA:A ratio $>1$ are given in table 3. The pulmonary artery diameter was not included in the hazard analysis due to its correlation with PA:A ratio $(r=0.62, \mathrm{p}<0.001)$. The analysis showed that a PA:A ratio $>1$ was a significant independent predictor of worse outcomes in both unadjusted and adjusted analyses (hazard ratio 3.99, 95\% CI 1.92-8.28, p<0.001 and hazard ratio 3.35, 95\% CI 1.54-7.26, $\mathrm{p}=0.002$, respectively). The GAP index was not statistically significant in either the unadjusted or adjusted analyses.

\section{Discussion}

In this study, we demonstrated that a PA:A ratio $>1$ is associated with worse outcomes in patients with IPF. Interestingly, the PA:A ratio $>1$ appeared to perform better in predicting outcomes than the well-established GAP index in our analysis.

In patients with IPF, accurate prognostication is crucial in determining further medical management, timing of evaluation and listing for lung transplantation, as well as for patient counselling. Currently, the determination of prognosis involves the use of clinical and physiological parameters such as age, gender and pulmonary function tests. Radiologic features that are used to predict outcomes in IPF are limited to the extent of fibrosis. The ability to use the PA:A ratio measurements obtained from readily available HRCT images in patients with IPF provides further important and complimentary prognostication data. The

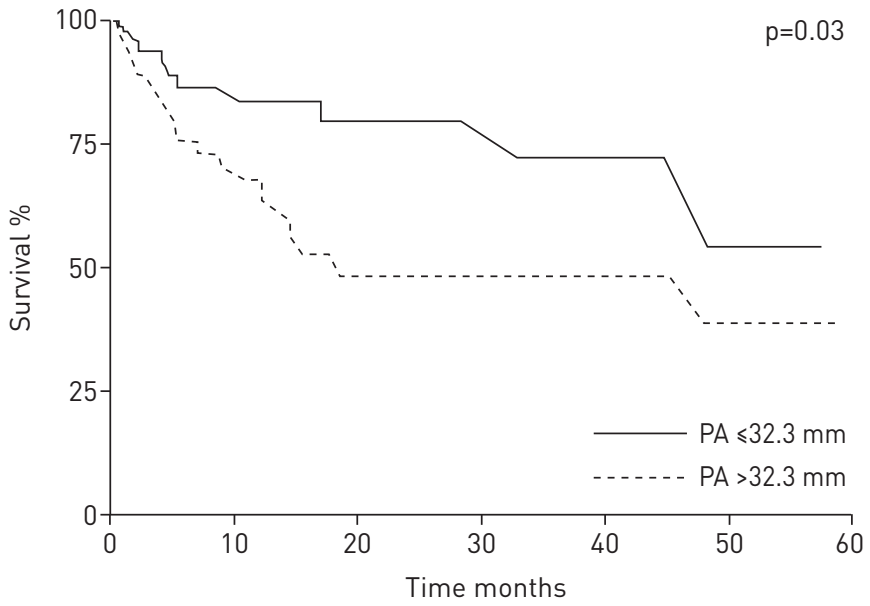

FIGURE 1 Transplant-free survival of the idiopathic pulmonary fibrosis cohort stratified by median pulmonary artery diameter (PA) $\leqslant 32.3 \mathrm{~mm}(\mathrm{n}=51)$ or $>32.3 \mathrm{~mm}(\mathrm{n}=47)$. 
FIGURE 2 Transplant-free survival of the idiopathic pulmonary fibrosis cohort stratified by pulmonary artery:ascending aorta diameter (PA:A) ratio $\leqslant 1(n=67)$ or $>1(n=31)$.

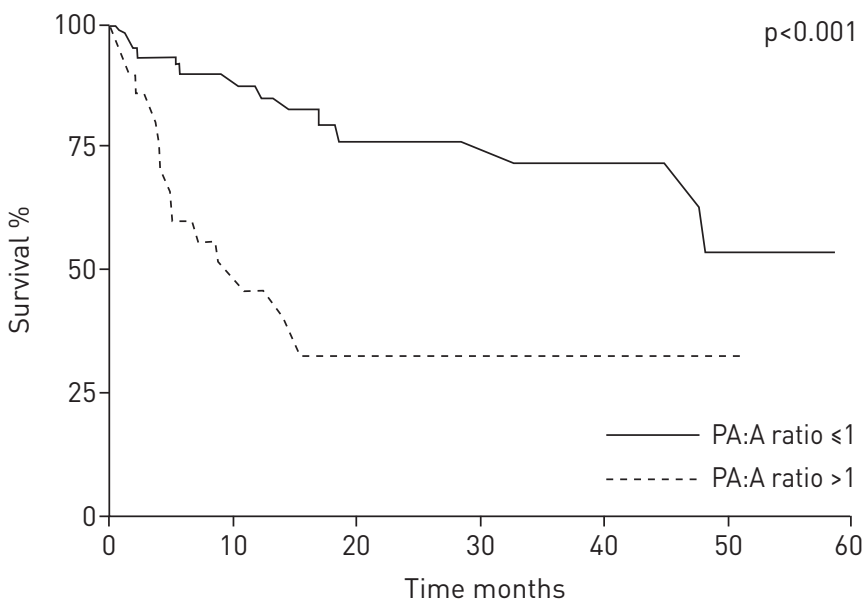

excellent intra-class correlation between our three readers supports that evaluating this ratio is not only easy, but also reliably consistent between readers and therefore readily applicable in the clinical setting.

There are several possible explanations for our observation of worse outcomes with a higher PA:A ratio. The association of the PA:A ratio with pulmonary hypertension has been reported in prior studies of IPF [11]. Specifically, it is well established that pulmonary hypertension is associated with worse outcomes in IPF, which may explain our observations [20]. Furthermore, prior studies have shown an association between PA:A ratio $>1$ with COPD exacerbations and worsened outcomes $[15,16]$. Whether or not the same relationship between exacerbations and pulmonary artery size holds true in IPF requires exploration in future studies. It is also noteworthy that an association between acute exacerbations and pulmonary hypertension has been reported in IPF [21]. Therefore, it is conceivable that some of the worse outcomes observed in our study may have also been due to acute exacerbations of IPF. The relationship between pulmonary artery size and pulmonary hypertension may not solely explain the worsened outcomes noted, and an increase in the PA:A ratio may be due to other reasons that are surrogates of disease severity. Prior studies comparing pulmonary artery size to pulmonary haemodynamics have also produced mixed results. This might suggest that factors other than pulmonary haemodynamics may play roles in the association between pulmonary artery size and outcomes. For example, it is plausible that the pulmonary artery size is affected by radial traction created by progressive fibrosis over time. Indeed, our analysis of pulmonary artery size and the PA:A ratio in relation to the FVC \% predicted and total lung capacity \% predicted demonstrated a weak negative correlation in our cohort, suggesting that the fibrosis itself may also play a role in pulmonary artery dilation.

An unintended consequence of our study is that we were able to provide an estimate of the prevalence of aortic aneurysms in patients with IPF. To the best of our knowledge, there have been no such reports previously, although it is well established that IPF patients have a greater propensity for cardiovascular events, including thromboembolic disease, coronary artery disease and heart failure [22-24]. The prevalence of aortic aneurysms in our IPF cohort was $6 \%$, which is slightly higher numerically than that of the general population (reported to be 4.6\%) [25]. Furthermore, a US-based study reported an overall age-

TABLE 3 Unadjusted and adjusted hazard ratios for lung transplant or death ${ }^{\#}$

\begin{tabular}{|c|c|c|c|c|}
\hline & \multicolumn{2}{|c|}{ Unadjusted analysis } & \multicolumn{2}{|c|}{ Adjusted analysis } \\
\hline & Hazard ratio $(95 \% \mathrm{CI})$ & p-value & Hazard ratio $(95 \% \mathrm{CI})$ & p-value \\
\hline FVC \% pred & $0.95(0.93-0.98)$ & $<0.001$ & & \\
\hline DLco $\%$ pred & $0.94(0.91-0.97)$ & $<0.001$ & & \\
\hline$B M I^{7} \mathrm{~kg} \cdot \mathrm{m}^{-2}$ & $0.99(0.92-1.06)$ & 0.73 & $0.98(0.91-1.05)$ & 0.61 \\
\hline $\mathrm{PA}: \mathrm{A}$ ratio $>1$ & $3.99(1.92-8.28)$ & $<0.001$ & $3.35(1.54-7.26)$ & 0.002 \\
\hline GAP index & $1.32(1.04-1.68)$ & 0.20 & $1.22(0.95-1.57)$ & 0.12 \\
\hline
\end{tabular}

FVC: forced vital capacity; DLCO: diffusing capacity of the lung for carbon monoxide; BMI: body mass index; PA:A ratio: pulmonary artery to ascending aorta diameter ratio; GAP index: scoring system based on gender, age and two lung physiology variables (FVC and $D\left\llcorner C 0\right.$ ). ${ }^{\#}: n=98$ patients; ${ }^{\text {" : }}$ BMI missing patient data $n=4$. 
and gender-adjusted incidence of thoracic aortic aneurysm as 10.4 per 100000 person-years [26]. This suggestion of an increased prevalence of aortic aneurysms among IPF patient requires further investigation in larger epidemiologic cohorts.

The limitations in our study include the variability that exists with capturing the pulmonary artery exactly at the level of bifurcation on the HRCT images. The very good correlation among our three readers, however, suggests that this is not a major issue. The presence of aortic aneurysmal enlargement might also affect the calculation of the ratio. However, only a small minority of our patients had aortic dilation/ aneurysm, which may have "normalised" their ratios. We chose to normalise the pulmonary artery size by the use of PA:A ratio only, as there is precedent for this in multiple other clinical trials $[16,27,28]$. In addition, in a prior large cohort study, in which the measurement of pulmonary artery size and PA:A ratio were stratified by various characteristics, including age, gender, height, body surface area and cardiovascular risk factors, only a weak to very weak correlation was demonstrated [29].

We were limited in our assessment of the correlation between pulmonary artery size and haemodynamic variables due to the number of available RHCs and their temporal relationship to HRCT. The available RHC data that was analysed supports a positive correlation between the MPAP and PA:A ratio with a notable stronger relationship with more temporally proximate HRCT studies. The paucity of contemporaneous haemodynamic data does not diminish from the powerful prognostic information imparted, but rather underscores the universal availability and applicability of this simple HRCT measurement. Specifically, we demonstrate that the PA:A ratio is a noninvasive tool that can be utilised as an independent prognostic indicator regardless of the status of concomitant pulmonary hypertension. Our finding of the PA:A ratio performing better than the GAP index should be viewed with some caution and requires further validation in a larger, independent cohort. One difference between our analysis of outcomes and the original GAP description is that ours was a composite of time to death or transplant, while the original description was as a predictor of mortality only.

In conclusion, measurement of the PA:A ratio on HRCT can be easily performed without additional invasive testing. A PA:A ratio $>1$ appears to be an independent predictor of outcomes in IPF patients. Evaluation of this readily available ratio may facilitate prognostication and counselling of patients with IPF. In addition, it may prove to have a role in stratifying patients within clinical studies as well as prioritisation for lung transplantation. Future studies are encouraged to further evaluate this ratio. Such studies might include correlating the ratio with contemporaneous invasive haemodynamic measurements as well as looking at the significance of change in the pulmonary artery diameter and PA:A ratio over time.

\section{References}

1 American Thoracic Society/European Respiratory Society. International multidisciplinary consensus classifications of the idiopathic interstitial pneumonias. Am J Respir Crit Care Med 2002; 165: 277-304.

2 Barlo NP, van Moorsel CH, van den Bosch JM, et al. Predicting prognosis in idiopathic pulmonary fibrosis. Sarcoidosis Vasc Diffuse Lung Dis 2010; 27: 85-95.

3 Ley B, Ryerson CJ, Vittinghoff E, et al. A multidimensional index and staging system for idiopathic pulmonary fibrosis. Ann Intern Med 2012; 156: 684-691.

4 Nathan SD, Basavaraj A, Reichner C, et al. Prevalence and impact of coronary artery disease in idiopathic pulmonary fibrosis. Respirology 2010; 104: 1035-1041.

5 Rivera-Lebron BN, Forfia PR, Kreider M, et al. Echocardiographic and hemodynamic predictors of mortality in idiopathic pulmonary fibrosis. Chest 2013; 144: 564-570.

6 King TE Jr, Tooze JA, Schwarz MI, et al. Predicting survival in idiopathic pulmonary fibrosis: scoring system and survival model. Am J Respir Crit Care Med 2001; 164: 1171-1181.

7 Lettieri CJ, Nathan SD, Barnett SD, et al. Prevalence and outcomes of pulmonary arterial hypertension in advanced idiopathic pulmonary fibrosis. Chest 2006; 129: 746-752.

8 Weill D, Benden C, Corris PA, et al. A consensus document for the selection of lung transplant candidates: 2014 - an update from the Pulmonary Transplantation Council of the International Society for Heart and Lung Transplantation. J Heart Lung Transplant 2015; 34: 1-15.

9 Nathan SD, Shlobin OA, Barnett SD, et al. Right ventricular systolic pressure by echocardiography as a predictor of pulmonary hypertension in idiopathic pulmonary fibrosis. Respir Med 2008; 102: 1305-1310.

10 Arcasoy SM, Christie JD, Ferrari VA, et al. Echocardiographic assessment of pulmonary hypertension in patient with advanced lung disease. Am J Respir Crit Care Med 2003; 167: 735-740.

11 Devaraj A, Wells AU, Meister MG, et al. The effect of diffuse pulmonary fibrosis on the reliability of CT signs of pulmonary hypertension. Radiology 2008; 249: 1042-1049.

12 Zisman DA, Karlamangla AS, Ross DJ, et al. High-resolution chest CT findings do not predict the presence of pulmonary hypertension in advanced idiopathic pulmonary fibrosis. Chest 2007; 132: 773-779.

13 McCall RK, Ravenel JG, Nietert PJ, et al. Relationship of main pulmonary artery diameter to pulmonary arterial pressure in scleroderma patients with and without interstitial fibrosis. J Comput Assist Tomogr 2014; 38: 163-168.

14 Tan RT, Kuzo R, Goodman LR, et al. Utility of CT scan evaluation for predicting pulmonary hypertension in patients with parenchymal lung disease. Medical College of Wisconsin Lung Transplant Group. Chest 1998; 113: $1250-1256$.

15 Shin S, King CS, Brown AW, et al. Pulmonary artery size as a predictor of pulmonary hypertension and outcomes in patients with chronic obstructive pulmonary disease. Respir Med 2014; 108: 1626-1632. 
16 Wells JM, Washko GR, Han MK, et al. Pulmonary arterial enlargement and acute exacerbations of COPD. $N$ Engl J Med 2012; 367: 913-921

17 American Thoracic Society/European Respiratory Society. Idiopathic pulmonary fibrosis: diagnosis and treatment. International consensus statement. Am J Respir Crit Care Med 2000; 161: 646-664.

18 Raghu G, Collard HR, Egan JJ, et al. An official ATS/ERS/JRS/ALAT statement: idiopathic pulmonary fibrosis: evidence-based guidelines for diagnosis and management. Am J Respir Crit Care Med 2011; 183: 788-824.

19 Mao SS, Ahmadi N, Shah B, et al. Normal thoracic aorta diameter on cardiac computed tomography in healthy asymptomatic adults: impact of age and gender. Acad Radiol 2008; 15: 827-834.

20 Adir Y, Harai S. Pulmonary hypertension associated with chronic obstructive lung disease and idiopathic fibrosis. Curr Opin Pulm Med 2014; 20: 414-420.

21 Judge EP, Fabre A, Adamali HI, et al. Acute exacerbations and pulmonary hypertension in advanced idiopathic pulmonary fibrosis. Eur Respir J 2012; 40: 93-100.

22 Sprunger DB, Olson AL, Huie TJ, et al. Pulmonary fibrosis is associated with an elevated risk of thromboembolic disease. Eur Respir J 2012; 39: 125-132.

23 Kizer JR, Zisman DA, Blumenthal NP, et al. Association between pulmonary fibrosis and coronary artery disease. Arch Intern Med 2004; 164: 551-556.

24 Han MK, McLaughlin V, Criner GJ, et al. Cardiovascular involvement in general medicine conditions. Circulation 2007; 16: 2992-3005.

25 Palmieri V, Bella JN, Arnett DK, et al. Aortic root dilation at sinuses of valsalva and aortic regurgitation in hypertensive and normotensive subjects: the Hypertension Genetic Epidemiology Network Study. Hypertension 2001; 37: 1229-1235.

26 Olsson C, Thelin S, Stahle E, et al. Thoracic aortic aneurysm and dissection: increasing prevalence and improved outcomes reported in a nationwide population-based study of more than 14,000 cases from 1987 to 2002 . Circulation 2006; 114: 2611-2618.

27 Chen X, Liu K, Wang Z, et al. Computed tomography measurement of pulmonary artery for diagnosis of COPD and its comorbidity pulmonary hypertension. Int J Chron Obstruct Pulmon Dis 2015; 10: 2525-2533.

28 Ng CS, Wells AU, Padley SP. A CT sign of chronic pulmonary arterial hypertension: the ratio of main pulmonary artery to aortic diameter. J Thorac Imaging 1999; 14: 270-278.

29 Truong QA, Massaro JM Rogers IS, et al. Reference values for normal pulmonary artery dimensions by noncontrast cardiac computed tomography: the Framingham Heart Study. Circ Cardiovasc Imaging 2012; 5: $147-154$. 